\title{
Uso autónomo de recursos de Internet entre estudiantes de ingeniería como fuente de ayuda matemática
}

\section{Autonomous Use of Internet Resources among Engineering Students as a Source of Mathematical Help-seeking}

\author{
Danelly S. Esparza Puga ${ }^{1}$
}

Resumen. Se presenta un estudio enfocado en analizar el uso autónomo de recursos de Internet por parte de estudiantes de ingeniería como fuente de ayuda para sus clases de matemáticas. Se utiliza un método cualitativo basado en entrevistas individuales y grupos focales. Los resultados muestran que los estudiantes recurren frecuentemente a recursos como YouTube, Facebook y Google para buscar ayuda matemática; particularmente, los consultan para aclarar dudas y repasar temas vistos en clase, encontrar distintas vías para resolver un problema, comprobar resultados y ver paso a paso soluciones de problemas resueltos. Los estudiantes reportan beneficios de usar estas fuentes de ayuda como el acceso a demasiada información sin límites ni de tiempo ni geográficos, pero también encuentran ciertos inconvenientes, como las restricciones de acceso debidas a derechos de autor y la presencia de elementos que pueden alejarlos del foco de búsqueda debido a la gran cantidad de información disponible y distractores tales como las redes sociales. Se concluye señalando algunas limitaciones del estudio y posibles rutas de investigación futura.

Fecha de recepción: 3 de diciembre de 2016. Fecha de aceptación: 3 de septiembre de 2017

1 Universidad Autónoma de Ciudad Juárez. Instituto de Ingeniería y Tecnología (IIT). Departamento de Física y Matemáticas. danelly.esparza@uacj.mx 
Palabras clave: búsqueda de ayuda; educación superior; Internet; redes sociales; educación matemática.

\begin{abstract}
Study that focus on analysing the autonomous use of Internet resources by engineering students as a source of mathematical help-seeking for their lessons. It was used a qualitative method based on individual interviews and focal groups. The results show that students often turn to sites like YouTube, Facebook and Google to find mathematical help; particularly, they resort to these sites to clarify doubts and review topics covered in class, to find different ways to solve a problem, verify results, and to see task solutions explained step by step. The students report benefits of using these help sources such as access to almost unlimited information without time or geographical restrictions, but they also find limitations like access restrictions due to copyright, and the presence of elements that can get them away from the search focus because the wealth of information available and distractors such as social networks. The report concludes by pointing out some limitations of the study and possible paths for future research.
\end{abstract}

Keywords: Help-seeking; higher education; Internet; social networks; mathematics education.

\title{
I. INTRODUCCIÓN Y ESTADO DE LA CUESTIÓN
}

Matemáticas es una asignatura escolar en la cual es muy común que los alumnos busquen ayuda cuando tienen dudas relacionadas con los temas estudiados. Es tan común que sería difícil imaginar a una persona que haya estudiado matemáticas en la escuela y que jamás recurriera a sus compañeros de clase, a algún familiar, o a su profesor(a) para obtener ayuda y tratar de aclarar sus dudas matemáticas; no obstante, como se ilustrará más adelante, las tecnologías digitales como Internet y los dispositivos móviles están modificando la manera en que los estudiantes buscan ayuda e información para clarificar las inquietudes relacionadas con sus lecciones de matemáticas. Sin embargo, hasta el momento no se ha estudiado lo suficiente cómo las prácticas de búsqueda de ayuda matemática se están modificando debido a la presencia de estos agentes tecnológicos. 
La búsqueda de ayuda es un área de investigación bien establecida que durante décadas ha capturado la atención de sociólogos, psicólogos sociales y educadores, entre otros especialistas; puede ser entendida como el proceso de buscar recursos externos a uno mismo para encontrar información o estrategias que ayuden a completar una tarea o resolver un problema; es una estrategia de aprendizaje auto regulada. En un principio fue conceptualizada como una actividad no deseable, ya que era entendida como un indicador de falta de independencia en el individuo, que podía generar costos para su autoestima y su sentido de competitividad (Shapiro, 1978). Los trabajos de Nelson-Le Gall (1985) contribuyeron a cambiar la conceptualización de la búsqueda de ayuda en el área de educación al proponer que podía ser vista como una habilidad deseable para los alumnos, pues les permitiría abordar y resolver problemas que serían difíciles de solventar por ellos mismos. La búsqueda de ayuda podía ser interpretada como una habilidad útil para el autoaprendizaje.

Según Nelson-Le Gall (1981) existen al menos dos tipos de búsqueda de ayuda: la instrumental y la ejecutiva. La ejecutiva se refiere a instancias en las que la intención del estudiante es conseguir que alguien resuelva su problema, pero sin interesarse en conocer la manera en que se soluciona; es una búsqueda de ayuda en la que el sujeto busca soluciones finalizadas y definitivas que le permitan reducir el tiempo y esfuerzo que le demandaría resolver por sí mismo el problema. En cambio, la búsqueda de ayuda instrumental está orientada hacia el dominio de la tarea y se refiere a instancias en las que el pedido de ayuda se focaliza en adquirir procesos exitosos de resolución de problemas. En este caso, la asistencia solicitada suele limitarse a solicitar sugerencias o las pistas necesarias para poder resolver el problema por uno mismo.

Los estudios pedagógicos más recientes sobre búsqueda de ayuda se han enfocado en indagar las maneras en que tecnologías como Internet, los ambientes de aprendizaje interactivo o los dispositivos móviles están impactando en las prácticas de búsqueda de ayuda de los alumnos (ver, por ejemplo, Karabenick y Puustinen, 2013). No obstante estos avances en la investigación educativa sobre el tema, en el área de la educación matemática poco se ha estudiado acerca de cómo las tecnologías digitales están afectando la forma en que los estudiantes contemporáneos buscan ayuda para resolver sus dudas y tareas matemáticas de forma independiente -es decir, sin la presencia de un profesor o investigador-, como apoyo para aclarar sus dudas en matemáticas o resolver sus tareas. 
Esto no quiere decir que el campo de la educación matemática esté alejado del estudio de las tecnologías como Internet y los dispositivos móviles. Existen compendios de investigaciones recientes que abordan cómo las tecnologías móviles pueden ser utilizadas en la enseñanza y el aprendizaje de las matemáticas (Crompton y Traxler, 2015; Meletiou-Mavrotheris, Mavrou y Paparistodemou, 2015). En el caso del uso de Internet en la enseñanza de las matemáticas, durante años ha sido un tema de constante interés en el campo; sin embargo, la mayoría de los estudios desarrollados se han centrado en investigar cómo emplear Internet para proveer instrucción matemática a los estudiantes y oportunidades de desarrollo profesional a los docentes de matemáticas (Engelbrecht y Harding, 2005; Borba et al., 2016; Borba y Llinares, 2012).

Por otro lado, algunas investigaciones han evidenciado cómo los estudiantes contemporáneos utilizan recursos de Internet, las redes sociales y los dispositivos móviles para buscar ayuda que apoye sus estudios en matemáticas. Por ejemplo, Van de Sande (2011) analizó un foro gratuito, abierto y en línea, al que miles de estudiantes de todo el mundo recurren en busca de ayuda para resolver sus dudas y tareas matemáticas (http://mathhelpforum.com). Este fue uno de los primeros estudios sobre búsqueda de ayuda matemática en Internet, el cual puso en evidencia no solo que los estudiantes de distintas regiones del mundo utilizaban los foros en Internet como fuente de apoyo, sino que también evidenció que las formas de buscar ayuda variaban de usuario a usuario, identificando algunas más productivas para el aprendizaje matemático.

Otro estudio -también enfocado en el análisis de un foro en línea de ayuda para resolver tareas matemáticas- es el de Puustinen, Bernicot, Volckaert-Legrier y Baker (2015), quienes se centraron en analizar los mensajes intercambiados entre los estudiantes que solicitaban ayuda y los profesores de matemáticas que fungían como voluntarios para contestar las dudas matemáticas. Los investigadores identificaron características en esos mensajes que facilitaban o dificultaban el trabajo de los profesores; por ejemplo, el proveer asistencia se facilitaba cuando el estudiante manifestaba una actitud de aprendizaje auto regulado al solicitar ayuda, como comunicar el trabajo preliminar que había hecho sobre la tarea matemática antes de requerir apoyo, o formular de manera explícita la solicitud de ayuda requerida.

No todos los estudios sobre búsqueda de ayuda matemática en Internet se han centrado en analizar foros en línea. En el trabajo de Cardoso, Kato y Oliveira (2014) se analiza la manera en que estudiantes utilizan un canal educativo de YouTube denominado "v13dinei", el cual se especializa en la 
publicación de videos con contenido matemático. Los investigadores encuentran que los videos relacionados con la asignatura de álgebra lineal son los más populares, en contraste con los de las asignaturas de cálculo u otras materias básicas de matemáticas. Entre los tópicos más consultados están principalmente la multiplicación de matrices y el cálculo de determinantes. Los resultados también muestran que el acceso a dicho canal se incrementa especialmente durante el periodo de evaluaciones escolares, lo cual podría indicar que estos videos son utilizados por los estudiantes para complementar sus estudios.

Por otro lado, el análisis de Aguilar y Puga (2015) expone la forma en la cual los dispositivos móviles (teléfonos celulares, tabletas y computadoras portátiles) son utilizados como fuente de ayuda matemática entre estudiantes universitarios. Los dispositivos son usados, por ejemplo, cuando el profesor plantea alguna pregunta a la clase y el alumno busca la respuesta en Internet; otros estudiantes utilizan las aplicaciones matemáticas para dispositivos móviles (como calculadoras con capacidades algebraicas) para comprobar cálculos o verificar respuestas.

Así, aunque algunos reportes de investigación evidencian que la nueva generación de alumnos recurre a las tecnologías digitales como fuente de ayuda para sus clases de matemáticas, nuestro conocimiento sobre la manera en que las utilizan sigue siendo limitado. Por ello, el principal propósito de este estudio es indagar interrogantes relacionadas con el uso de Internet como proveedora de ayuda matemática entre estudiantes de ingeniería, sin limitarse al estudio de fuentes específicas; en particular, el interés es investigar lo siguiente: 1) ¿Cuáles son los recursos de Internet más utilizados como fuente de ayuda para sus clases de matemáticas?, 2) ¿Cuál es la frecuencia con la que los utilizan?, 3) ¿Cuáles son las potencialidades y limitaciones que perciben del uso de Internet en sus estudios matemáticos?, y 4) ¿Cuáles son las características de los sitios web que los hacen confiables?, todo desde el punto de vista de los estudiantes.

\section{METODOLOGÍA}

Este fue un estudio de corte cualitativo compuesto por dos fases, una exploratoria y otra final. Se iniciará describiendo la fase exploratoria -que sirvió para probar el funcionamiento del cuestionario diseñado con el propósito de recolectar las opiniones de los estudiantes-, se presentará y discutirá ese cuestionario y también se detallará el contexto en el cual fue desarrollado el estudio, así como las 
características de los entrevistados. Finalmente, se explicará cómo fueron recolectados y analizados los datos empíricos.

\section{FASE EXPLORATORIA}

El estudio inició con una fase exploratoria, en la cual se aplicó un cuestionario de siete preguntas abiertas que sirvieron de guión para llevar a cabo entrevistas semiestructuradas. Los objetivos de esta fase fueron los siguientes: 1) Confirmar que los estudiantes universitarios utilizan Internet cuando tienen dudas o necesitan ayuda en sus clases de matemáticas, y 2) Evaluar si el cuestionario estaba adecuadamente diseñado para generar los datos empíricos requeridos; por ejemplo, si la formulación de las preguntas era clara y si las respuestas obtenidas proporcionaban información útil para responder las inquietudes de investigación planteadas.

En la fase exploratoria se entrevistaron estudiantes del Instituto de Ingeniería y Tecnología (IIT) de la Universidad Autónoma de Ciudad Juárez (UACJ), en México. Es la universidad pública más importante de Ciudad Juárez, con una población aproximada de 26,405 estudiantes distribuidos en 116 programas educativos en cuatro institutos; se trata de una institución pública de nivel superior localizada al norte de México, en la frontera con El Paso, Texas, en los Estados Unidos de América. El presente estudio se llevó a cabo en el Instituto de Ingeniería y Tecnología, el cual alberga aproximadamente 5,620 alumnos y cuenta con 14 carreras de licenciatura (ingenierías), todas diseñadas para cursar la totalidad de los créditos en nueve semestres. Durante los primeros cuatro semestres los estudiantes toman asignaturas básicas de matemáticas como cálculo diferencial, integral y vectorial, álgebra lineal, ecuaciones diferenciales, análisis vectorial, probabilidad y estadística, entre otras.

Los alumnos entrevistados en esta fase y en la fase final debían cumplir principalmente con dos aspectos: que fueran alumnos de alguna de las ingenierías de la UACJ y que al momento de las entrevistas estuvieran inscritos al menos en una asignatura del área de matemáticas, esto con la intención de garantizar que tuvieran alguna experiencia reciente sobre búsqueda de ayuda en sus clases de matemáticas. Así, tomando en cuenta los aspectos mencionados, se entabló una plática sobre el presente estudio con un grupo de estudiantes de ingeniería en la cual fueron expuestos los objetivos de la investigación y se les aclaró que, si decidían participar, sus opiniones serían utilizadas 
únicamente con fines de investigación, precisando también que su participación en el proyecto sería anónima. Los entrevistados que decidieron participar de forma voluntaria en el proyecto cumplían con los requisitos mencionados: eran estudiantes de ingeniería de la UACJ y en ese momento cursaban las asignaturas de cálculo integral, álgebra lineal y ecuaciones diferenciales. Con la finalidad de resguardar toda la información, cada una de las entrevistas fue audio grabada para su posterior análisis.

En la fase exploratoria, inicialmente fueron entrevistados de forma individual cuatro estudiantes del IIT; eran universitarios de sexo masculino provenientes de tres carreras: ingeniería eléctrica, ingeniería industrial e ingeniería en sistemas digitales. Cuando se realizaron las entrevistas uno de ellos cursaba el tercer semestre, otro estaba en cuarto y dos cursaban el primer semestre de su carrera; fueron alumnos de diferentes niveles. En el presente estudio se consideran alumnos de nivel principiante aquellos que estén en primero, segundo y tercer semestre; de nivel intermedio quienes estudien los semestres cuarto, quinto y sexto; finalmente, son de nivel avanzado los que estén en séptimo, octavo o noveno. Las edades de los cuatro entrevistados eran 21, 23, 25 y 38 años, respectivamente.

El instrumento que se utilizó fue un cuestionario, el cual funcionó como guía para obtener las opiniones de los participantes. Las preguntas que lo constituyen son las siguientes:

1. ¿Has utilizado algún recurso de Internet para tu clase de matemáticas?

2. ¿Cuáles recursos has utilizado para tus clases de matemáticas?

3. ¿Con qué frecuencia utilizas estos recursos?

4. ¿De qué forma utilizas estos recursos de Internet en tus estudios de matemáticas?

5. Menciona los beneficios de utilizar recursos de Internet en tu formación matemática en la universidad.

6. Menciona las limitaciones que tiene el utilizar recursos de Internet en tu formación matemática como estudiante universitario.

7. En tu opinión, ċqué recurso de Internet es más confiable y qué lo hace confiable?

El rol de la primera pregunta del cuestionario fue conocer si los estudiantes universitarios recurren a recursos de Internet para sus clases de matemáticas. La pregunta número dos fue diseñada para indagar cuáles recursos son los más 
consultados por los estudiantes de ingeniería entrevistados cuando requieren ayuda matemática. La intención de la tercera pregunta fue investigar la frecuencia de tiempo con la que se utilizan los recursos mencionados en la pregunta anterior, es decir, si su uso era diario, semanal, mensual, etcétera. El propósito de la pregunta cuatro era tratar de entender de qué manera los alumnos utilizan Internet como fuente de ayuda para sus clases de matemáticas. Las preguntas cinco y seis estuvieron enfocadas en identificar los beneficios y limitaciones percibidos al utilizar Internet como recurso de ayuda. Finalmente, la última pregunta estuvo dirigida a explorar las razones o características que un recurso de Internet debe tener para que los estudiantes lo perciban como una fuente confiable.

Con base en los resultados obtenidos en la fase exploratoria, fue posible confirmar que los alumnos universitarios participantes utilizan variados recursos de Internet como fuente de ayuda para sus clases de matemáticas. En su totalidad, los cuatro expresaron utilizar Internet como recurso para sus clases de matemáticas, siendo la red social YouTube el sitio más mencionado; también mencionaron sitios que ofrecen Cursos Masivos Abiertos en Línea (mooc por sus siglas en inglés), como los del Instituto de Tecnología de Massachusetts (mit) (http://www. ocw.mitedu/courses) o aquellos ofrecidos por el sitio edX (http://www.edx.org).

Después de obtener los datos de esta fase, se consideró que el instrumento era adecuado para continuar con la fase final de la investigación.

\section{FASE FINAL}

Las entrevistas de la fase final se dividieron en dos partes. En la primera se entrevistó individualmente a cinco estudiantes de nivel intermedio, es decir, del cuarto y quinto semestres, quienes cursaban en ese momento la asignatura de cálculo vectorial; las entrevistas fueron audio grabadas.

En esta primera parte los entrevistados eran alumnos de ambos sexos provenientes de tres distintas carreras: ingeniería eléctrica, ingeniería biomédica e ingeniería civil. Cuando se efectuaron las entrevistas tres de ellos cursaban cuarto semestre y los otros dos el quinto semestre de sus respectivas carreras; sus edades variaban entre los 19 y 23 años. En este primer conjunto de entrevistas se realizaron encuentros individuales en los cuales, entre otras cosas, se percibió que los alumnos se cohibían al momento de dar respuesta a las preguntas, y se limitaban a decir breves palabras. Fue por esa razón que se optó por hacer una toma de datos adicional, pero con 
un método distinto. En la toma de datos adicional se formó un grupo focal con seis sujetos (grupo focal 1), donde la mayoría eran estudiantes de nivel avanzado, situados entre los semestres séptimo y onceavo de ingeniería industrial y manufactura. A diferencia de cuando los fueron entrevistados individualmente, ahora aparentaban sentirse más cómodos al expresar sus opiniones y compartir sus experiencias, por estar rodeados de sus compañeros en el grupo que se había formado.

La literatura muestra cómo los entrevistados que son tímidos logran expresarse abiertamente cuando se encuentran en grupos focales: hacen gestos, movimientos y dan respuestas concretas, a diferencia de cuando son entrevistados individualmente. Esto quiere decir que sienten un ambiente de confianza cuando se encuentran rodeados de compañeros que comparten sus experiencias, al igual que ellos. Asimismo, la intención de los grupos focales fue promover la auto-apertura entre los entrevistados, ya que a algunos individuos, la auto-exposición para expresar sus ideas frente a otras personas les resulta fácil, natural y cómoda, mientras que a otros es difícil e incómoda (Ho, 2006; Krueger y Casey, 2008; Morgan, 1990).

Debido al evidente cambio de actitud entre los participantes entrevistados en el grupo focal 1, se decidió realizar entrevistas en un segundo grupo focal constituido por seis estudiantes (grupo focal 2). Este grupo fue constituido por alumnos de los semestres intermedios de ingeniería en mecatrónica. La participación de los entrevistados también fue voluntaria y sus respuestas fueron audio grabadas para su posterior análisis.

\section{ANÁLISIS DE RESULTADOS}

Para llevar a cabo el análisis de los datos generados se hicieron transcripciones de las entrevistas y posteriormente se efectuó un análisis clásico de contenido (Onwuegbuzie, Dickinson, Leech y Zoran, 2009), esto es, se crearon pequeños grupos de datos (extractos de las transcripciones), se les asignaron códigos que correspondían con las respuestas a las preguntas del cuestionario, y fueron contabilizados todos los casos que correspondían a un mismo código. Los códigos empleados fueron: recursos utilizados, frecuencia de uso, forma de uso, beneficios y limitaciones percibidas, sitios considerados confiables, razones para confiar en los recursos. Los resultados mostrados a continuación provienen del análisis de las 17 entrevistas realizadas a los participantes en la fase final del estudio. 


\section{FRECUENCIA DE USO DE LOS SITIOS DE INTERNET COMO FUENTE DE AYUDA MATEMÁTICA}

Los datos indican que la totalidad de los entrevistados declaró utilizar algún recurso de Internet como fuente de ayuda para sus clases de matemáticas. Se elaboró una clasificación de los tipos de usuarios según las respuestas obtenidas: usuarios frecuentes y usuarios medianos. Los usuarios frecuentes son aquellos que mencionan utilizar Internet diariamente con propósitos escolares, y los usuarios medianos son quienes utilizan estos recursos los fines de semana, cuando tienen tiempo libre.

Después del análisis de los datos, es notorio que la mayoría de los estudiantes son usuarios frecuentes ya que de los 17 entrevistados, 12 afirmaron utilizar estos recursos diariamente y solo 5 universitarios mencionaron que utilizan este tipo de recursos cada fin de semana, argumentando que durante la semana no tienen tiempo debido a sus actividades diarias, como el hecho de desempeñar algún trabajo después o antes de llegar a la escuela.

\section{RECURSOS DE INTERNET MÁS POPULARES Y SU FUNCIONALIDAD}

En la Figura 1 se muestra la frecuencia con que fueron mencionados algunos recursos de Internet referidos como fuente de ayuda matemática por los estudiantes que participaron en el estudio.

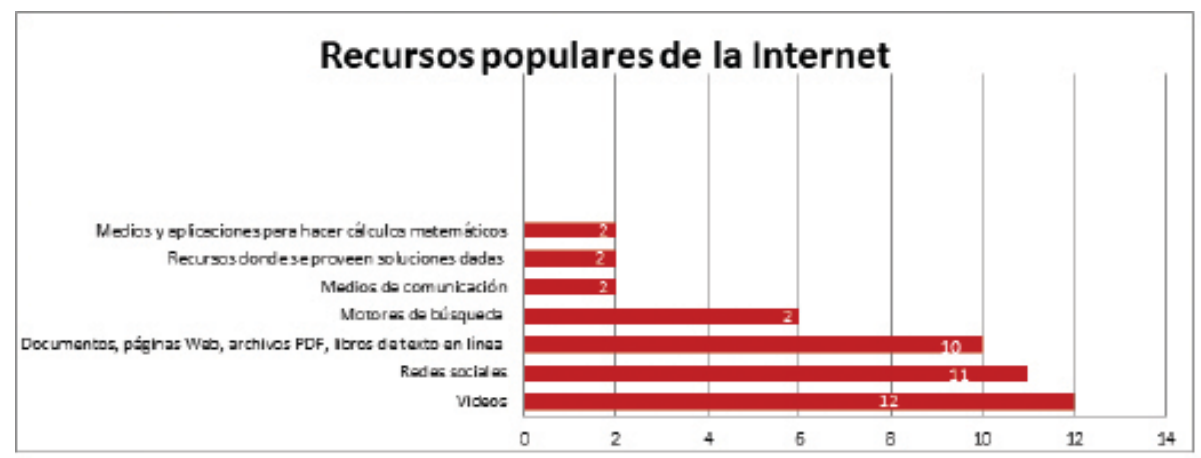

Figura 1. Recursos de Internet más frecuentemente referidos como fuente de ayuda matemática. 
El recurso de Internet más citado fue YouTube, sitio web dedicado a compartir videos. Doce de los 17 entrevistados coinciden en que YouTube es el sitio al cual recurren para comprender mejor los temas vistos en clase, como lo ilustra la siguiente afirmación de uno de los estudiantes:

Estudiante 1, grupo focal 2: "YouTube es un sitio visual y si no comprendo la explicación de un video puedo ver muchos otros... veo un video y luego otro y es totalmente diferente, pero me lleva al mismo resultado"

En la segunda posición se encuentran las redes sociales como Facebook, pues 11 de los 17 entrevistados lo han utilizado para sus clases de matemáticas. Los estudiantes aseveran que Facebook es una herramienta que, en su mayoría, utilizan para contactar con compañeros de clase o con el maestro cuando les surgen dudas sobre trabajos, tareas o inquietudes de logística, por ejemplo conocer el aula donde se dictará una clase.

Estudiante 2, grupo focal 1: “... utilizo Facebook con mis compañeros cuando tengo dudas de lo que explican en clase, ya que en ocasiones cuando llego a casa no recuerdo lo que vi"

Otros recursos de Internet utilizados por los estudiantes de ingeniería son los documentos de texto, páginas web, archivos PDF y libros de texto en línea (10 menciones). Los siguientes extractos ilustran estos usos:

Estudiante 5, grupo focal 2: "Hay una página que se llama creo Vitutor.com ahí vienen muchos ejercicios de muchas materias... problemas resueltos de matemáticas y para hacer [...] te da todo paso por paso".

Estudiante 5, entrevista individual: "Me gusta mucho PDF porque siento que son más seguros que Wikipedia y así..."

Estudiante 1, entrevista individual: “... Uno que se llama Web Profesores es una página donde los profesores suben documentos o técnicas de enseñanza [...] son documentos escritos [...] una vez metí una fórmula que necesitaba en Google y así llegué a la página y también hay otra que es lo mismo... Profesores Universia" 
En la cuarta posición se encuentran los motores de búsqueda, con seis menciones. A pesar de existir numerosos buscadores como Google, Bing, Yahoo, Aol Search y AltaVista, entre otros, Google es el único motor de búsqueda citado en el presente estudio. En Google es donde los alumnos participantes buscan directamente los temas que desean investigar y donde algunos realizan búsquedas un poco más especializadas haciendo uso de Google Libros, Google Académico o estrategias para delimitar la información y mejorar las búsquedas, es decir, para restringir los sitios, documentos o formatos donde quieren enfocar su búsqueda.

Estudiante 3, grupo focal 2: "Yo casi lo primero que hago es buscar en Google el tema, y ya voy viendo cuál se me hace más fácil de entender"

Estudiante 4, grupo focal 1: "Yo pongo en Google... no sé... la ecuación punto pendiente y luego pongo filetype, dos puntos PDF [ecuación punto pendiente filetype:pdf] y manda todo en PDF"

A pesar de que no todos los alumnos declaran utilizar buscadores, pareciera que el primer paso cuando buscan ayuda en Internet es recurrir a los motores de búsqueda; inicialmente escriben el tema en cuestión o algunas palabras clave, y posteriormente seleccionan la información. Por ejemplo, el estudiante 4 del grupo focal 1 hace uso de una estrategia para restringir su búsqueda: menciona que al agregar la expresión "filetype:PDF" delimita los resultados a documentos que se encuentren únicamente en formato pdf ya que, en su opinión, le generan mayor confianza.

En quinta posición se encuentran medios de comunicación (dos menciones), como lo es el correo electrónico, fuente utilizada entre los estudiantes para comunicarse principalmente con sus maestros. En la sexta posición están los recursos donde se proveen soluciones dadas (dos menciones), por ejemplo Yahoo respuestas:

Estudiante 4, entrevista individual: "A veces ya ve que le dan un problema y las preguntas ya están subidas [sic... Yahoo respuestas lo utilizaría para ya cuando batallas y dices ay no..."

Estudiante 1, grupo focal 1: "Busco ejercicios hechos, nada más lo googleo y el resultado que aparezca" 
En la séptima y última posición de los recursos de Internet más utilizados se encuentran medios y aplicaciones para hacer cálculos matemáticos, específicamente hicieron dos referencias a Wolfram Alpha, sitio basado en uno de los programas creados por Wolfram Research Mathematica, que incorpora el procesamiento de álgebra, cálculo numérico y simbólico, visualizaciones y capacidades estadísticas. Los estudiantes hacen referencia a este sitio cuando intentan comprobar resultados, ya sea de derivadas o integrales:

Estudiante 2, grupo focal 2: “... Utilizo Wolfram Alpha para puros resultados, pero en veces [sic] no es muy confiable por que salta unos pasos [...] nada más metes la ecuación y ya te da paso por paso..."

Haciendo referencia a lo anterior, se puede concluir que la mayoría de los recursos de Internet a los que acuden los alumnos de ingeniería que participaron en este estudio se utilizan para: 1) Aclarar dudas y reforzar temas vistos en clase, 2) Encontrar distintas vías para solucionar un problema, 3) Comprobar resultados, y 4) Ver soluciones de problemas hechas paso a paso.

\section{BENEFICIOS Y LIMITACIONES PERCIBIDAS POR LOS ESTUDIANTES}

Algunos de los entrevistados destacan que una de las ventajas es el acceso geográfico y temporal ilimitado, esto quiere decir que consideran importante el hecho de tener acceso a Internet en cualquier lugar y a cualquier hora. El siguiente extracto de una entrevista individual ilustra este punto:

Estudiante 5, entrevista individual: "Yo pienso que ahorro de... por ejemplo si debo ir a la biblioteca tengo que manejar... ahorro tiempo y dinero"

Otro beneficio mencionado es que encuentran útil Internet para aclarar dudas, ya que existen infinidad de páginas en las cuales pueden consultar. Hay quien la percibe como una fuente de ayuda infalible:

Estudiante 3, grupo focal 2: "El beneficio que encuentro es que cualquier cosa que busque la voy a encontrar, son miles de páginas las que van a salir y siempre encontraré algo, nunca me quedaré con la duda" 
Sin embargo, el beneficio más aludido entre los entrevistados es el hecho de que por este medio encuentran distintas vías para solucionar problemas:

Estudiante 1, grupo focal 1: "Cuando entro a YouTube... tengo la opción de ver distintas formas de solucionar un problema, si no entiendo un video, voy a otro y así hasta que logro entender"

Estudiante 4, grupo focal 2: "En veces [sic] no entiendo cómo resolvió el problema el maestro y busco otra forma de solucionarlo"

En suma, los beneficios percibidos por los estudiantes al usar Internet como fuente de ayuda matemática son: 1) El acceso sin limitaciones geográficas o de tiempo, 2) La infinidad de fuentes de información disponibles, y 3) La posibilidad de encontrar maneras distintas y alternativas de abordar problemas matemáticos.

En cuanto a las limitaciones percibidas sobre el uso de Internet como fuente de ayuda matemática para sus estudios, se detectó que algunos alumnos de semestres avanzados de ingeniería no encuentran beneficios en el uso de sitios populares de Internet, ya que no encuentran material especializado en su área de estudio. Por otro lado, algunos estudiantes consideran como limitación el acceso restringido por derechos de autor y/o idioma, como lo mencionan en los siguientes comentarios:

Estudiante 5, grupo focal 1: “... Yo... lo mismo que comenté, encontrar algo más específico, buen material en español casi no encuentras..."

Estudiante 6, grupo focal 2: "El problema es que si no sabes lo que estás buscando recibes respuestas muy ambiguas, información muy ambigua y en la Internet se regulan mucho los derechos de autor, si quieres leer un libro solo puedes ver una parte [...] el material útil está restringido"

Otras limitaciones manifestadas fueron, por un lado, la gran cantidad de información disponible en Internet, que puede provocar que el estudiante se pierda; por otro lado, los múltiples distractores a los que se enfrentan, por ejemplo las redes sociales:

Estudiante 5, grupo focal 2: “A mí me pasó una vez que estudiando para un examen había un tema que de plano no sabía nada [...] encontré un método que nada que ver, que no era lo que necesitaba [...] a veces pones algo y salen como mil cosas de eso, no es lo que andas buscando" 
Estudiante 2, grupo focal 1: "Cuando estoy haciendo tareas me distraigo con mucha facilidad revisando Facebook o viendo videos en YouTube"

Para resumir, las limitaciones que perciben los participantes sobre el uso de Internet como fuente de ayuda matemática son: 1) El acceso restringido por derechos de autor y/o idioma, 2) El exceso de información disponible, y 3) Los distractores que pueden encontrar al estar buscando ayuda.

\section{SITIOS CONSIDERADOS CONFIABLES Y RAZONES PARA CONFIAR EN ELLOS}

Algunos de los estudiantes que participaron en este estudio basan la confiabilidad de las fuentes de ayuda que obtienen de Internet en el prestigio o autoridad que puede brindar un grado o una posición académica, o bien una institución. Por ejemplo:

Estudiante 1, entrevista individual: “... Profesores Universia porque está avalado por varias universidades latinoamericanas"

Estudiante 3, grupo focal 1: "Por ejemplo, en Youtu [sic], bueno ahí los videos son de los profesores de universidades y ellos mismos hacen los videos para aclarar las dudas, para mí es muy confiable porque son profesores de universidades..."

Estudiante 5, entrevista individual: “... SlideShare se me hace más confiable porque ahí los doctores mandan sus presentaciones"

Así, los estudiantes entrevistados califican de confiables a sitios como Profesores Universia, YouTube y SlideShare. Las razones por las que los consideran confiables están relacionadas 1) Con el prestigio o estatus de la institución educativa que refrenda los sitios consultados, y 2) Con el grado académico o nombramiento de los autores de los materiales consultados.

\section{DISCUSIÓN DE RESULTADOS Y CONCLUSIONES}

El presente estudio estuvo enfocado en estudiar la manera en que Internet es utilizada de manera autónoma entre estudiantes de ingeniería cuando necesitan 
ayuda para sus clases de matemáticas. Todos los participantes en el estudio declararon utilizar Internet como apoyo para sus clases de matemáticas, y es utilizado diariamente por la mayoría de ellos.

Entre los recursos de Internet más frecuentemente mencionados como fuente de ayuda matemática se encuentran los videos (YouTube) y las redes sociales (Facebook). Estos resultados coinciden con las observaciones de Cardoso et al. (2014), que señalan a YouTube como una fuente de ayuda matemática popular entre estudiantes de países iberoamericanos. Este resultado concuerda también con los señalamientos de Goodband, Solomon, Samuels, Lawson y Bhakta (2012), en el sentido de que Facebook puede fungir como un espacio que promueva la comunicación matemática dentro de comunidades de estudiantes universitarios. Asimismo, estos resultados añaden evidencia de cómo Facebook puede funcionar como un espacio para el aprendizaje informal que apoye el aprendizaje de los contenidos vistos en el aula.

Por otra parte, se identificaron algunas de las razones por las que los alumnos recurren a estos sitios: aclarar dudas y repasar temas vistos en clase, encontrar distintas vías para resolver un problema, comprobar resultados y ver soluciones de problemas elaboradas paso a paso.

Las declaraciones de los estudiantes sugieren que algunos buscan ayuda de tipo instrumental, en la que se interesan no solo en obtener la solución a los problemas sino también entender -paso a paso- cómo resolverlos, o incluso conocer maneras alternativas de solución. Por otro lado, también hay evidencia de alumnos que parecen enfocarse en conseguir una ayuda de tipo ejecutiva en la que la respuesta a los problemas está dada, como la estudiante 4 de la entrevista individual al decir que usa el sitio Yahoo Respuestas cuando no quiere batallar. Sin embargo, la información obtenida en la presente investigación no es suficiente para afirmar de manera definitiva el tipo de búsqueda de ayuda que están realizando los estudiantes. Actualmente se está trabajando en profundizar lo anterior, así como indagar en las materias y los tópicos de matemáticas más consultados entre estudiantes de ingeniería.

Resultados similares son reportados en Van de Sande (2011), donde se identifican distintos tipos de comportamientos en los alumnos que buscan ayuda matemática en foros abiertos en Internet; por ejemplo algunos de ellos ingresan al foro solamente a plantear sus dudas, esperando que sean resueltas sin interactuar con los demás miembros, mientras que otros solicitan ayuda de forma más activa, interactuando de manera más rica y significativa con los proveedores de ayuda matemática. 
Acerca de los beneficios percibidos al usar Internet como fuente de ayuda matemática, destacan el acceso a información casi ilimitada, sin restricciones de tiempo ni geográficas, lo cual posibilita encontrar distintas maneras de abordar problemas matemáticos. En cuanto a las limitaciones detectadas, algunos estudiantes mencionan las restricciones de acceso debido a derechos de autor e idioma, así como elementos que pueden alejarte del foco de búsqueda, por ejemplo la gran cantidad de información disponible y la presencia de distractores como las redes sociales.

En relación a los sitios que consideran confiables para buscar ayuda matemática, fueron mencionados principalmente Profesores Universia, YouTube y SlideShare; sin embargo, cuando se trata de evaluar la confiabilidad de estos sitios, los estudiantes parecen no poner atención a las propiedades matemáticas intrínsecas de la información allí obtenida (Lithner, 2003), sino que basan su evaluación en características de la información que no guardan relación con las matemáticas, tales como el prestigio académico de la persona o institución que publica la información.

Una de las limitaciones de este estudio es que está basado en auto reportes sobre el uso de tecnología, lo cual puede ser menos preciso que las observaciones de los comportamientos reales de los estudiantes. Una futura ruta de investigación se podría enfocar en utilizar métodos de observación más directa como el propuesto por Junco (2014), donde se utilice software en los dispositivos empleados por los estudiantes, para así documentar y caracterizar los comportamientos de búsqueda de ayuda manifestados cuando utilizan distintos dispositivos como computadoras, tabletas y teléfonos móviles.

Otra posible ruta de investigación sobre la búsqueda de ayuda matemática sería incluir la perspectiva de los profesores de matemáticas sobre este fenómeno educativo. Por ejemplo, ¿qué piensan ellos de esta práctica de sus estudiantes?, ¿la consideran deseable o provechosa?, ¿integran este tipo de fuentes de ayuda en la instrucción matemática que proveen a sus estudiantes? Este tipo de estudios ayudaría a contribuir en el conocimiento que se tiene acerca de la manera en que los profesores de matemáticas perciben y promueven los comportamientos de búsqueda de ayuda de sus alumnos (Marais, Van der Westhuizen y Tillema, 2013).

En síntesis, el estudio de las prácticas de búsqueda de ayuda matemática entre los estudiantes contemporáneos es de gran relevancia no solo para la investigación educativa sino también para la instrucción matemática, ya que estas prácticas se encuentran ampliamente difundidas y debemos entenderlas con el propósito de poder visualizar sus posibles potencialidades y riesgos para la educación matemática. 


\section{REFERENCIAS}

Aguilar, M. S. \& Puga, D. E. (2015). Mobile Help-seeking in Mathematics: An Exploratory Study with Mexican Engineering Students. In: H. Crompton \& J. Traxler (eds.). Mobile Learning in Mathematics: Foundations, Design, and Case Studies (176-186). New York: Routledge.

Borba, M. C. \& Llinares, S. (2012). Online Mathematics Teacher Education: Overview of an Emergent Field of Research. ZDM Mathematics Education, 44(6), 697-704. DOI: 10.1007/s11858-012-0457-3.

Borba, M. C., Askar, P., Engelbrecht, J., Gadanidis, G., Llinares, S. \& Aguilar, M. S. (2016). Blended Learning, e-learning and Mobile Learning in Mathematics Education. ZDM Mathematics Education, 48(5), 589-610. DOI: 10.1007/s11858-016-0798-4.

Cardoso, V. C., Kato, L. A. \& Oliveira, S. R. (2014). Where to Learn Math? A Study of Access to an Educational Channel on YouTube. International Journal for Research in Mathematics Education, 4(3). Recuperado de http://www.sbembrasil.org.br/ripem/index.php/ ripem/article/view/92/100.

Crompton, H. \& Traxler, J. (eds.). (2015). Mobile Learning and Mathematics. Foundations, Design and Case Studies. Florence: Routledge.

Engelbrecht, J. \& Harding, A. (2005). Teaching Undergraduate Mathematics on the Internet. Part 1: Technologies and Taxonomy. Educational Studies in Mathematics, 58(2), 235-252. DOI: 10.1007/s10649-005-6456-3.

Goodband, J. H., Solomon, Y., Samuels, P. C., Lawson, D. \& Bhakta, R. (2012). Limits and Potentials of Social Networking in Academia: Case Study of the Evolution of a Mathematics Facebook Community. Learning, Media and Technology, 37(3), 236-252. DOI: 10.1080/17439884.2011.587435.

Ho, D. (2006). The Focus Group Interview: Rising to the Challenge in Qualitative Research Methodology. Australian Review of Applied Linguistics, 29(1), 1-19. DOl: 10.2104/ aral0605.

Junco, R. (2014). iSpy: Seeing what Students Really Do Online. Learning, Media and Technology, 39(1), 75-89. DOI: 10.1080/17439884.2013.771782.

Karabenick, S. A. \& Puustinen, M. (eds.). (2013). Advances in Help-seeking Research and Applications: The Role of Emerging Technologies. Charlotte, North Carolina: Information Age Publishing.

Krueger, R. \& Casey, M. (2008). Focus Groups: A Practical Guide for Applied Research. London: Sage.

Lithner, J. (2003). Students' Mathematical Reasoning in University Textbook Exercises. Educational Studies in Mathematics, 52(1), 29-55. DOI: 10.1023/A:1023683716659. 
Marais, C., Van der Westhuizen, G. \& Tillema, H. (2013). Teacher Knowledge of Learners' Help-seeking in Mathematics Problem Solving. African Journal of Research in Mathematics, Science and Technology Education, 17(1-2), 63-73. DOl: http://dx.doi.org/10.1 080/10288457.2013.826971.

Morgan, D. (1990). Focus Groups as Qualitative Research. London: Sage.

Nelson-Le Gall, S. (1981). Help-seeking: An Understudied Problem-solving Skill in Children. Developmental Review, 1(3), 224-246. DOI: 10.1016/0273-2297(81)90019-8.

Nelson-Le Gall, S. (1985). Help-seeking Behavior in Learning. Review of Research in Education, 12(1), 55-90. DOI: 10.3102/0091732X012001055.

Onwuegbuzie, A. J., Dickinson, W. B., Leech, N. L. \& Zoran, A. G. (2009). A Qualitative Framework for Collecting and Analyzing Data in Focus Group Research. International Journal of Qualitative Methods, 8(3), 1-21. DOl: 10.1177/160940690900800301.

Puustinen, M., Bernicot, J., Volckaert-Legrier, O. \& Baker, M. (2015). Naturally Occurring Help-seeking Exchanges on a Homework Help Forum. Computers \& Education, 81, 89-201. DOI: 10.1016/j.compedu.2014.09.010.

Shapiro, E. G. (1978). Help-seeking: Effects of Visibility of Task Performance and Seeking Help. Journal of Applied Social Psychology, 8(2), 163-173. DOI: 10.1111/j.15591816.1978.tb00774.x.

Van de Sande, C. (2011). A Description and Characterization of Student Activity in an Open, Online, Mathematics Help Forum. Educational Studies in Mathematics, 77(1), 53-78. DOI: 10.1007/s10649-011-9300-y. 
